\title{
AN APPROXIMATION CONNECTED WITH THE EXPONENTIAL FUNCTION
}

\author{
K. SONI AND R. P. SONI
}

(Communicated by J. Marshall Ash)

\begin{abstract}
Some recent techniques in the uniform asymptotic expansions of integrals are used to obtain an expansion for a function related to the exponential function. This function is associated with Ramanujan, Watson, Copson, and Buckholtz. The results obtained complement those given by Buckholtz.
\end{abstract}

\section{INTRODUCTION}

Let

$$
\frac{(n z)^{n}}{n !} S_{n}(z)=e^{n z}-\sum_{k=0}^{n} \frac{(n z)^{k}}{k !} .
$$

In 1963 Buckholtz [3] gave an asymptotic expansion of $S_{n}(z)$ that is valid in the whole complex $z$-plane except $z=1$. He proved that if $z \neq 1$, then

$$
S_{n}(z) \sim n !\left(\frac{e^{z}}{n z}\right)^{n} \phi_{K}(z)+\sum_{k=0}^{\infty} n^{-k} u_{k}(z),
$$

where

$$
u_{k}(z)=\left(\frac{z}{z-1} \frac{d}{d z}\right)^{k} \frac{z}{1-z}=\frac{(-1)^{k+1} Q_{k}(z)}{(z-1)^{2 k+1}},
$$

$Q_{k}(z)$ is a polynomial of degree $k$ with positive integer coefficients, and $\phi_{K}(z)$ is the characteristic function of the set $K=\left\{z /|z| \geq 1\right.$ and $\left.\left|z e^{1-z}\right| \leq 1\right\}$, see Figure 1. Furthermore, Buckholtz proved that this expansion holds uniformly in the region where $d_{K}(z) \geq \varepsilon>0, d_{K}(z)$ being the distance from $z$ to the set $K$. In particular, for $z=-1$, it yields result of Copson [4]. As $z \rightarrow 1$, each of the coefficients $u_{k}(z)$ in (1.2) become unbounded. On the other hand,

$$
S_{n}(1)=\frac{n !}{2}\left(\frac{2}{3}\right)^{n}-\frac{2}{3}+\frac{4}{135 n}+O\left(\frac{1}{n^{2}}\right), \quad n \rightarrow \infty .
$$

This approximation of $S_{n}(1)$ is associated with a very elegant result communicated by Ramanujan to Hardy and later discussed at length by Watson [10].

Received by the editors April 7, 1989.

1991 Mathematics Subject Classification. Primary 41A60, 30E15. 


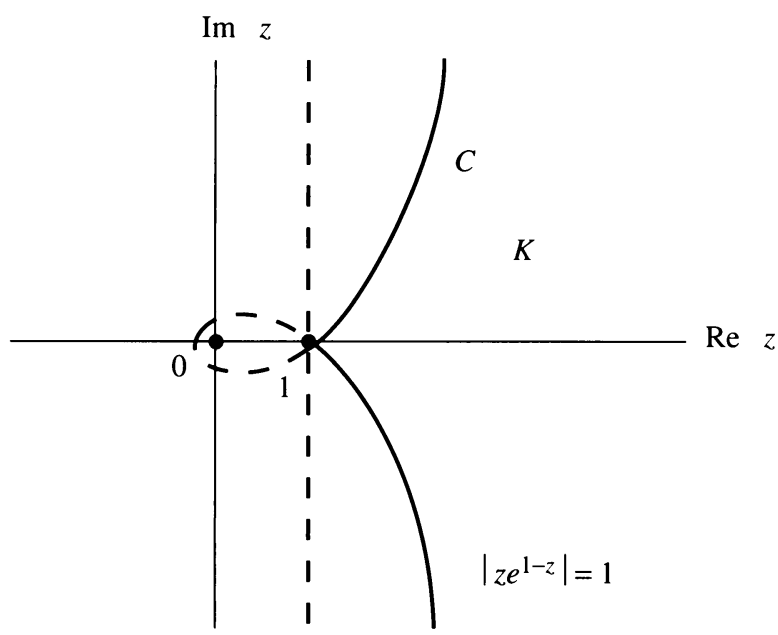

Figure 1. The $z$-PLANE.

Buckholtz remarks that in view of (1.2) and (1.3), Ramanujan's approximation is a considerably more singular result than it would otherwise appear. Apart from the fact that the expansion (1.2) is not valid at $z=1$, we note that it is also discontinuous across the curve $C$, the boundary of $K$ and that the curve $C$ passes through the point $z=1$.

In this note we obtain an asymptotic expansion of $S_{n}(z)$ that holds uniformly in a neighborhood of $z=1$. In particular, we investigate how the behavior of $S_{n}(z)$ changes across the curve $C$ in that neighborhood. By the foregoing discussion we should expect $z=1$ to be a singular point of the expansion. Consequently, the expansion is a generalized asymptotic expansion rather than an expansion of the Poincare type. As in Watson [10] and Wong [11], we start with an integral representation of $S_{n}(z)$. By using the integral form of the remainder,

$$
\begin{aligned}
(n z)^{n} S_{n}(z) & =\int_{0}^{n z}(n z-t)^{n} e^{t} d t \\
& =(n z)^{n+1} \int_{0}^{1}(1-u)^{n} e^{n z u} d u .
\end{aligned}
$$

By another change of variable,

$$
\frac{S_{n}(z)}{n z}=\int_{0}^{\infty} e^{-n\left(v-e^{\alpha}+e^{\alpha-v}\right)} e^{-v} d v, \quad z=e^{\alpha} .
$$

The change from the $z$ - to the $\alpha$-plane makes the discussion much simpler later on. The point $z=1$ corresponds to $\alpha=0$. Therefore, we want the asymptotic expansion of the integral (1.6) in the neighborhood of $\alpha=0$. Let

$$
\psi(v)=v-e^{\alpha}+e^{\alpha-v} .
$$

Since $\psi^{\prime}(v)=1-e^{\alpha-v}, \psi^{\prime \prime}(v)=e^{\alpha-v}$, it follows that $\psi(v)$ has a simple saddle point at $v=\alpha$. As $\alpha \rightarrow 0$, the two critical points $v=\alpha$ and $v=0$, that is, the saddle point and an end of the interval of integration, coalesce. A uniform asymptotic expansion of such an integral is obtained by first reducing 
it to the standard form given by Bleistein [2]. However, after the standard form has been achieved, different techniques can be used to obtain the asymptotic expansion (see, for example, [9-11]). We use a technique developed recently in $[7,8]$. This not only provides the expansion coefficients explicitly, but also simplifies the discussion of the nature of expansion.

We would like to emphasize two points regarding the asymptotic expansion given here. The first is that the singularity of the expansion is restricted to a single multiplicative factor; considering the fact that Bleistein's technique is applicable, this is not really surprising. The second is that the expansion holds for all $z$ except the negative real axis. In view of the expansion given by Buckholtz, this is rather surprising. In $\S 2$, after some preliminary discussion, we state the main result. The proof is given in $\S \S 3$ and 4 .

\section{MAIN RESUlt}

Before we can state our main result precisely, we need to reduce (1.6) to the standard form. Let

$$
\begin{gathered}
v-e^{\alpha}+e^{\alpha-v}=w^{2} / 2-b w, \\
b=\alpha\left\{2\left(e^{\alpha}-1-\alpha\right) / \alpha^{2}\right\}^{1 / 2} .
\end{gathered}
$$

In (2.2) the principal branch of the square root function is taken. Furthermore, $b$ is chosen so that the saddle point $v=\alpha$ in the $v$-plane corresponds to the saddle point $w=b$ in the $w$-plane. Now the mapping (2.1) can be given as

$$
w-b=(v-\alpha)\left\{\frac{2\left[e^{-(v-\alpha)}+(v-\alpha)-1\right]}{(v-\alpha)^{2}}\right\}^{1 / 2} \text {. }
$$

Again, we take the principal branch of the function. Thus $w-b \sim v-\alpha$ when $v \rightarrow \alpha$. By (2.1),

$$
\frac{d w}{d v}=\frac{1-e^{\alpha-v}}{w-b}
$$

The point $v=\alpha$ is a regular point of the transformation given by (2.3). In fact, it can be shown that the mapping $w=w(v-\alpha)$ is one to one and maps the strip $\left|I_{m}(v-\alpha)\right| \leq \pi$ conformally on to a certain region in the $w$-plane. Let

$$
V=V_{1}+i V_{2}=v-\alpha, \quad W=X+i Y=w-b,
$$

and let $D$ denote the domain $\left|I_{m} V\right|=\left|I_{m}(v-\alpha)\right| \leq \pi$. The image of $D$ in the $W$ and the $w$-plane is denoted by $D^{\prime}$ and $D^{\prime \prime}$ respectively, (see Figures 2,3 , and 4). The boundary of the domain $D, V_{2}=\pi$ and $V_{2}=-\pi$ are mapped onto the hyperbolic curves $X Y=\pi, Y>0$ and $X Y=-\pi, Y<0$ respectively in the $W$-plane. The path of integration in each plane is indicated by an arrow.

By (1.6) and the transformation (2.1),

$$
\begin{aligned}
\frac{S_{n}(z)}{n z} & =\int_{0}^{\infty+b} e^{-n\left(w^{2} / 2-b w\right)} e^{-v} \frac{d v}{d w} d w \\
& =\int_{0}^{\infty+b} e^{-n\left(w^{2} / 2-b w\right)} g(w) d w
\end{aligned}
$$




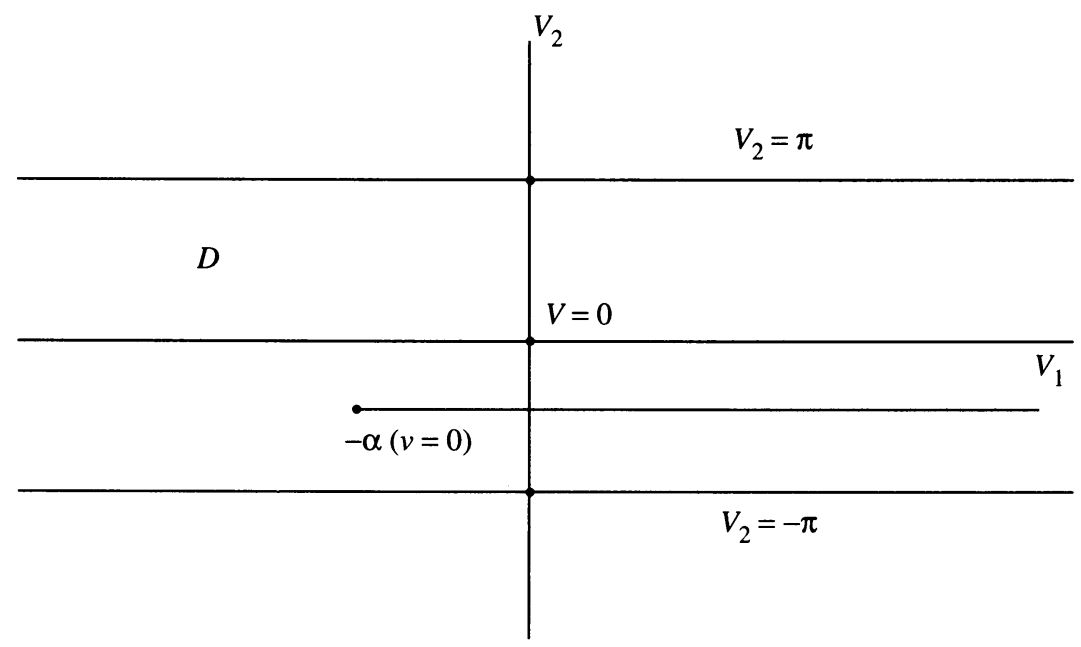

Figure 2. The $V$-Plane. $V=V_{1}+i V_{2}=v-\alpha$.

where

$$
g(w)=(w-b) /\left(e^{v}-e^{\alpha}\right)
$$

Since by (2.3),

$$
g(b)=\lim _{w \rightarrow b} g(w)=e^{-\alpha}=\frac{1}{z},
$$

we can write (2.6) as follows.

$$
(n z)^{-1} S_{n}(z)-\frac{1}{z} H_{n}(b)=\int_{0}^{\infty+b} e^{-n\left(w^{2} / 2-b w\right)} G(w)(w-b) d w
$$

where

$$
H_{n}(b)=\int_{0}^{\infty} e^{-n\left(w^{2} / 2-b w\right)} d w
$$

and

$$
G(w)=\{g(w)-g(b)\} /(w-b) .
$$

$H_{n}(b)$ is related to the complementary incomplete error function. The standard form of the integral mentioned earlier is given in (2.6), but (2.8) is more convenient for deriving the asymptotic expansion. $G(w)$ is analytic in the region, which in the $W=w-b$-plane, is shown as region $D^{\prime}$ (see Figure 3). Now we expand $G(w)$ in terms of the polynomials $P_{k}(w)$, which are defined as follows:

$$
\begin{aligned}
P_{0}(w)=1, & P_{1}(w)=w, \\
P_{k}^{\prime}(w)=(w-b) P_{k-2}(w), & P_{k}(0)=0, \quad k=2,3, \ldots .
\end{aligned}
$$

These polynomials are discussed in a more general form in [7, 8]. Since $G(w)$ is analytic in the region that includes the origin, we can write

$$
G(w)=\sum_{k=0}^{\infty} c_{k} P_{k}(w), \quad c_{k}=c_{k}(b) .
$$

This series converges in the neighborhood of the origin, at least for $|b|$ suffi- 


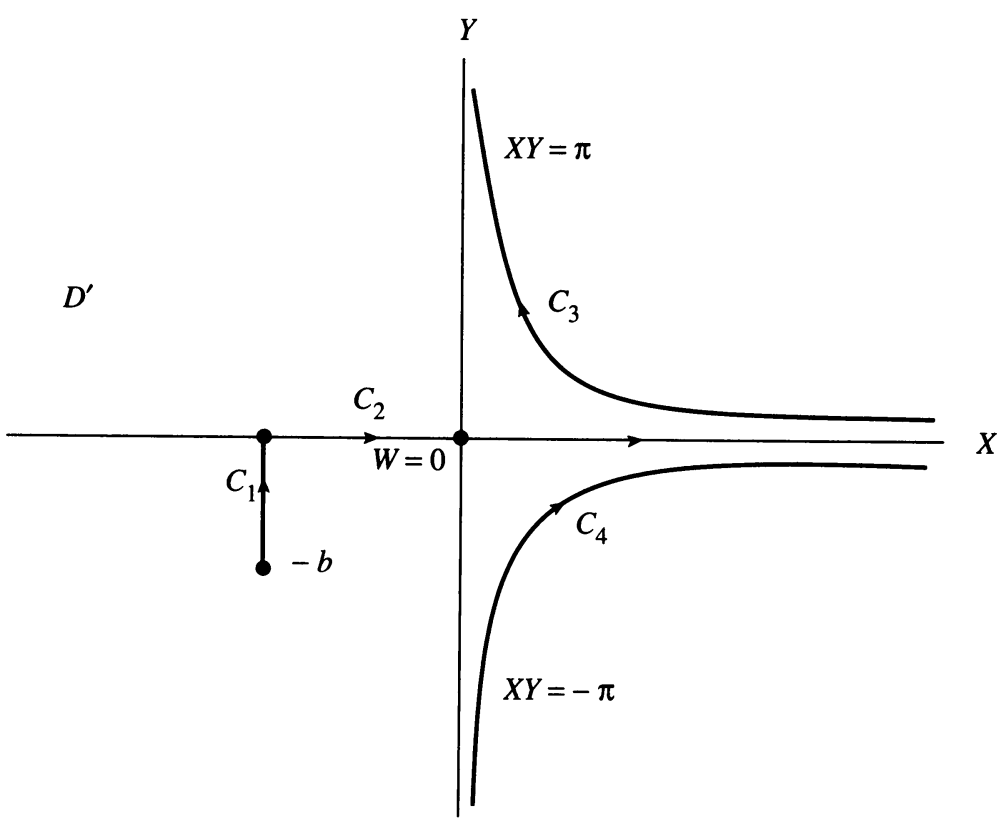

Figure 3. The $W$-PLANE.

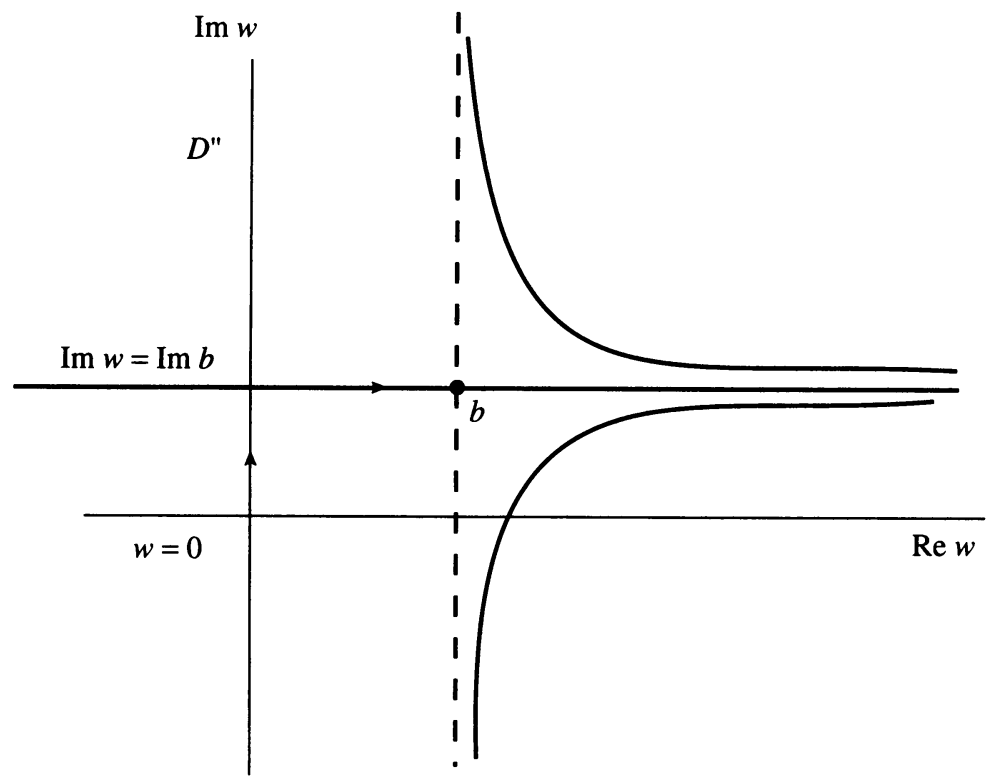

FiguRe 4. THE $w$-PLANE. $W=X+i Y=w-b,-b=$ $f(-\alpha)$.

ciently small. By using (2.11) and integration by parts successively, we see that for $k=0,1,2, \ldots$,

$$
\int_{0}^{\infty} e^{-n\left(w^{2} / 2-b w\right)} P_{2 k}(w)(w-b) d w=n^{-k-1},
$$




$$
\int_{0}^{\infty} e^{-n\left(w^{2} / 2-b w\right)} P_{2 k+1}(w)(w-b) d w=n^{-k-1} H_{n}(b)
$$

where $H_{n}(b)$ is defined by (2.9). Thus, by (2.8) and (2.12), we have the formal asymptotic expansion,

$$
(n z)^{-1} S_{n}(z) \sim\left(\frac{1}{z}+\sum_{k=0}^{\infty} \frac{c_{2 k+1}}{n^{k+1}}\right) H_{n}(b)+\sum_{k=0}^{\infty} \frac{c_{2 k}}{n^{k+1}} .
$$

Now we state the main result.

Theorem. Let $\Omega$ be a compact subset of the complex plane with the cut, $|\arg z|<$ $\pi,|z|>0$. Then for all $z \in \Omega$,

$$
(n z)^{-1} S_{n}(z)=\left(\frac{1}{z}+\sum_{k=0}^{N-1} \frac{c_{2 k+1}}{n^{k+1}}\right) H_{n}(b)+\sum_{k=0}^{N-1} \frac{c_{2 k}}{n^{k+1}}+E_{2 N}(z, n),
$$

$$
E_{2 N}(z, n)=\int_{0}^{\infty+b}\left[G(w)-\sum_{k=0}^{2 N-1} c_{k} P_{k}(w)\right](w-b) e^{-n\left(w^{2} / 2-b w\right)} d w
$$

$b=b(z)$ is determined uniquely by $z$ and satisfies

$$
e^{-b^{2} / 2}=z e^{1-z}
$$

Furthermore, (i) the coefficients $c_{k}$ are analytic functions of $z$ in $\Omega$, and (ii)

$$
E_{2 N}(z, n)=O\left(n^{-N} \max \left\{1,\left|H_{n}(b)\right|\right\}\right), \quad n \rightarrow \infty,
$$

uniformly in $\Omega$.

We will prove this in the next two sections. Here we would like to make a remark about the expansion. Since $c_{k}(b)$ are analytic functions of $z$, by (2.15) it is clear that the only singularity in the asymptotic expansion is due to the multiplicative factor $H_{n}(b)$. By [5, pp. $311-312 ; 1$, p. 300], $H_{n}(b)=O\left(n^{-1 / 2}\right)$ for $b=O\left(n^{-1 / 2}\right)$ and for $\left|b n^{1 / 2}\right| \gg 1$,

$$
H_{n}(b) \sim \begin{cases}e^{n b^{2} / 2}\left(\frac{2 \pi}{n}\right)^{1 / 2} & |\arg b|<\frac{\pi}{4}, \\ (-n b)^{-1} & \frac{\pi}{4} \leq|\arg b| \leq \pi .\end{cases}
$$

If $b=\sigma+i \tau$, the halflines $\sigma=\tau$ and $\sigma=-\tau, \sigma>0$ form the boundary of the region in which the part of the asymptotic expansion with $H_{n}(b)$ as a factor dominates. Note that by $(2.18),\left|e^{-b^{2} / 2}\right|=e^{-\left(\sigma^{2}-\tau^{2}\right) / 2}=\left|z e^{1-z}\right|$. Therefore the boundary $D$ of the region $K$ in the expansion given by Buckholtz, corresponds to the halflines $\sigma=|\tau|>0$.

\section{THE EXPANSION COEFFICIENTS}

Let $V=v-\alpha, W=w-b$ and

$$
f(\xi)=\xi\left\{2\left(e^{-\xi}+\xi-1\right) / \xi^{2}\right\}^{1 / 2}, \quad\left|I_{m}(\xi)\right| \leq \pi .
$$

By (2.3), $W=f(V), V \in D$ and $D^{\prime}=f(D)$. As remarked earlier, $f$ is analytic and one to one. Also, by (2.2), $b=-f(-\alpha), \alpha=\log z$. Therefore, 
$b$ is an analytic function of $z$ in $|\arg z|<\pi$ and mapping is one to one. If $z$ belongs to $\Omega$, then $-b$ is in the interior of $D^{\prime}$ and $b$ is in the interior of $D^{\prime \prime}$ (see Figure 4). To prove that $c_{k}$ are analytic functions of $z$, we only need to show that they are analytic functions of $b$. The $c_{k}$ are computed as follows (see [8, Theorem 5], $\gamma=0$ )

$$
c_{k}(b)=\frac{1}{2 \pi i} \int_{C_{w}} G(\zeta) \phi_{k}(\zeta) d \zeta,
$$

where $C_{w}$ is a simple, closed contour in the $w$-plane in the region $D^{\prime \prime}$ enclosing the points $w=0$ and $w=b$. The functions $\phi_{n}(\zeta)$ are generated by the expansion

$$
\frac{1}{\zeta-w}=\sum_{k=0}^{\infty} P_{k}(w) \phi_{n}(\zeta)
$$

They satisfy the following relation

$$
\phi_{0}(\zeta)=\frac{1}{\zeta}, \quad \phi_{1}(\zeta)=\frac{1}{(\zeta-b)^{2}}, \quad \phi_{k+2}(\zeta)=-\frac{d}{d \zeta} \frac{\phi_{k}(\zeta)}{(\zeta-b)} .
$$

The coefficients $c_{k}$ are computed by using the representations $[8,(3.1)$ and (3.2)],

$$
\begin{gathered}
\phi_{2 k-1}(\zeta)=\frac{(2 k) !}{k ! 2^{k}(\zeta-b)^{2 k}}, \\
\phi_{2 k}(\zeta)=\sum_{m=0}^{k} \frac{(k+m) !}{m ! 2^{m} \zeta^{k-m+1}(\zeta-b)^{k+m}} .
\end{gathered}
$$

We can choose the contour $C_{w}$ in $D^{\prime \prime}$ such that $\left\{b: b=-f(-\alpha), z=e^{\alpha}\right.$, $z \in \Omega\}$ and $w=0$ are inside it. By (2.7) and (2.10), $w=b$ is a regular point of $G$. Since

$$
G(w)=\left(e^{v}-e^{\alpha}\right)^{-1}-\frac{1}{z}(w-b)^{-1},
$$

the only singularities of $G$ are at the points that correspond to $v=\alpha+2 k \pi i$, $k= \pm 1, \pm 2 \ldots$. All such points are outside $D^{\prime \prime}$. Hence the analyticity of $c_{k}(b)$ follows from the representation (3.2). These coefficients can be obtained by the residue theorem. Near $v=\alpha$,

$$
\begin{aligned}
v-\alpha= & (w-b)+\frac{1}{6}(w-b)^{2}+\frac{1}{36}(w-b)^{3} \\
& +\frac{1}{270}(w-b)^{4}+\frac{1}{4320}(w-b)^{5}+\cdots
\end{aligned}
$$

and near $v=0$,

$$
v=b\left(e^{\alpha}-1\right)^{-1} w+2^{-1}\left(e^{\alpha}-1\right)^{-3}\left\{e^{\alpha} b^{2}-\left(e^{\alpha}-1\right)^{2}\right\} w^{2}+\cdots .
$$

The first four coefficients in the asymptotic expansion are,

$$
\begin{gathered}
c_{0}(b)=-\left(e^{\alpha}-1\right)^{-1}+\frac{e^{-\alpha}}{b}, \quad c_{1}(b)=\frac{e^{-\alpha}}{12}, \\
c_{2}(b)=\left(e^{\alpha}-1\right)^{-3}+e^{-\alpha}\left\{\frac{1}{12 b}-\frac{1}{b^{3}}\right\}, \quad c_{3}(b)=\frac{e^{-\alpha}}{288} .
\end{gathered}
$$


By using the expansion for $H_{n}(b)$ when $|\arg b|>\frac{\pi}{4}$, we obtain

$$
S_{n}(z)=\frac{z}{1-z}+\frac{1}{n} \frac{z}{(1-z)^{3}}+\cdots \text {. }
$$

These are the first two terms of Buckholtz's expansion. As $z \rightarrow 1, \alpha \rightarrow 0$ and therefore, $b \rightarrow 0$. By taking the limit as $\alpha \rightarrow 0, c_{0}(0)=-\frac{2}{3}, c_{1}(0)=\frac{1}{12}$, $c_{2}(0)=\frac{4}{135}$, and $c_{3}(0)=\frac{1}{288}$. Also $H_{n}(0)=\left(\frac{\pi}{2 n}\right)^{1 / 2}$. The expansion at $z=1$ reduces to

$$
S_{n}(1)=\left(\frac{\pi n}{2}\right)^{1 / 2}\left\{1+\frac{1}{12 n}+\frac{1}{288 n^{2}}\right\}-\frac{2}{3}+\frac{4}{135 n}+\cdots,
$$

which agrees with the form of $S_{n}(1)$ given in [3].

\section{THE REMAINDER $E_{2 N}$}

We will prove that the bound for $E_{2 N}(z, n)$ given in (2.19) holds uniformly in $z, z \in \Omega$. Without loss of generality we may assume that $\Omega=\{z /|\arg z| \leq$ $\pi-\delta$ and $\left.\Delta^{-1} \leq|z| \leq \Delta\right\}$. Then $|\operatorname{Re} \alpha| \leq \ln \Delta$ and $|\operatorname{Im} \alpha| \leq \pi-\delta$. Denote this set by $\Omega^{\prime}$ and let $\Omega^{\prime \prime}=f\left(\Omega^{\prime}\right)$ so that $-b$ belongs to $\Omega^{\prime \prime}$. Since $f$ maps the real $V$-axis onto the real $W$-axis, $\Omega^{\prime \prime}$ is symmetric about the real $W$-axis. Let

$$
R_{2 N}(w)=G(w)-\sum_{k=0}^{2 N-1} c_{k} P_{k}(w)
$$

Now we use some recent results concerning the polynomials $P_{k}$ and the associated functions $\phi_{k}$. By [7, (7.5) and (7.6) with $\left.\gamma=0\right],(2.17)$ can be written as follows:

$$
\begin{aligned}
& E_{2 N}(z, n)=n^{-N} \int_{0}^{\infty+b} \mathscr{D}^{N}\left[R_{2 N}(w)\right](w-b) e^{-n\left(w^{2} / 2-b w\right)} d w \\
& =n^{-N} e^{n b^{2} / 2} \int_{-b}^{\infty} \mathscr{D}^{N}\left[R_{2 N}(W+b)\right] W e^{-n W^{2} / 2} d W \\
& \mathscr{D} R_{2 N}(w)=(w-b)^{-1} \frac{d}{d w} R_{2 N}(w), \quad \mathscr{D}^{m}=\mathscr{D}^{m-1}, \quad m=2,3, \ldots
\end{aligned}
$$

We can replace the line of integration in the last integral by the contours $C_{1}$ and $C_{2}$ where $C_{1}$ is the straight line segment joining $-b=-\sigma-i \tau$ and $-\sigma+i 0$ and $C_{2}$ is the halfline $x=\operatorname{Re} W \geq-\sigma$. Let us assume for the present that $\mathscr{D}^{N} R_{2 N}$ is bounded on $C_{1}$ and $C_{2}$. Then

$$
\begin{aligned}
\int_{C_{1}} \mathscr{D}^{N} R_{2 N}(W+b) W e^{-n W^{2} / 2} d W & =O\left(\int_{0}^{\tau}\left(\sigma^{2}+t^{2}\right)^{1 / 2} e^{-n\left(\sigma^{2}-t^{2}\right) / 2} d t\right), \\
& =O\left(e^{-n\left(\sigma^{2}-\tau^{2}\right) / 2}\right), \quad n \rightarrow \infty
\end{aligned}
$$

Since $-b$ belongs to the compact set $\Omega^{\prime \prime}$, the implied constant in (4.3) can be 
taken independently of $\sigma$ and $\tau$. Again,

$$
\begin{aligned}
\int_{C_{2}} \mathscr{D}^{N} R_{2 N}(W+b) W e^{-n W^{2} / 2} d W & =O\left(\int_{-\sigma}^{\infty}|X| e^{-n X^{2} / 2} d X\right) \\
& = \begin{cases}O\left(\frac{1}{n}\right) & \sigma \geq 0, \\
O\left(\frac{1}{n} e^{-n \sigma^{2} / 2}\right) & \sigma<0 .\end{cases}
\end{aligned}
$$

In each case, the implied constant is independent of $b$. By (4.3) and (4.4) we obtain (2.19). To complete the proof we need to show that $\mathscr{D}^{N} R_{2 N}(W+b)$ is indeed bounded for all $W$ on $C_{1}$ and $C_{2}$. Again, we use the technique developed in [8]. By [8, (6.17)],

$$
\mathscr{D}^{N}\left[R_{2 N}(w)\right]=\frac{1}{2 \pi i} \int_{C_{w}} \Theta_{\zeta}^{N}(\zeta-w)^{-1} G(\zeta) d \zeta,
$$

where $C_{w}$ is a contour in the $w$-plane within $D^{\prime \prime}$ and enclosing the points $\zeta=$ $0, \zeta=b$, and $\zeta=w ; \boldsymbol{\Theta}_{\zeta}(\zeta-w)^{-1}=-\frac{d}{d \zeta}(\zeta-b)^{-1}(\zeta-w)^{-1}, \boldsymbol{\Theta}_{\zeta}^{m}=\boldsymbol{\Theta}_{\zeta} \boldsymbol{\Theta}_{\zeta}^{m-1}$. By $[8$, Lemma 3],

$$
\boldsymbol{\theta}_{\zeta}^{N}(\zeta-w)^{-1}=\sum_{m=0}^{N} \frac{(N+m) !}{m ! 2^{m}(\zeta-b)^{N+m}(\zeta-w)^{N-m+1}} .
$$

Therefore, by a change of variable,

$$
\mathscr{D}^{N}\left[R_{2 N}(W+b)\right]=\sum_{m=0}^{N} \frac{(N+m) !}{m ! 2^{m}} \int_{C_{W}} \frac{G(\eta+b)}{\eta^{N^{+} m}(\eta-W)^{N-m+1}} d \eta ;
$$

where $\eta=\zeta-b, W=w-b$, and $C_{W}$ is the contour in the $W$-plane corresponding to $C_{w}$ in the $w$-plane. Note that by (2.7) and (2.10),

$$
G(W+b)=\frac{1}{z}\left\{\frac{1}{e^{V}-1}-\frac{1}{W}\right\} \text {. }
$$

$G(W+b)$ is not only analytic in $D^{\prime}$, it is also bounded. On $V=V_{1} \pm i \pi$, $-\infty<V_{1}<\infty,\left(e^{V}-1\right)^{-1}$ is bounded. By the Phragmén-Lindelöf theorem and the maximum principle, $G(W(V)+b)=G(W+b)$ is bounded for $V \in D$ or $W \in D^{\prime}$. Now consider the boundaries of $D$ and $D^{\prime}$. The boundary of $D^{\prime}$ is the map of $V=V_{1} \pm i \pi$. Let $W=X+i Y$. By (2.3) and (3.1), $V=V_{1} \pm i \pi$,

$$
X^{2}-Y^{2}=2\left(-e^{-V_{1}}+V_{1}-1\right), \quad X Y= \pm \pi .
$$

The boundary curves of $D^{\prime}$ are, $X Y=\pi, Y>0$ and $X Y=-\pi, Y<0$. By considering the mapping (2.3), it follows that $V_{1} \rightarrow \infty$ if and only if $X \rightarrow \infty$. By (4.9), $V_{1} \sim X^{2} / 2, X \rightarrow \infty$. Therefore, on the curves $X Y= \pm \pi$,

$$
\left|\left(e^{V}-1\right)^{-1}\right|=\left(e^{V_{1}}+1\right)^{-1} \leq e^{-X^{2} / 4}, \quad X \gg 1 .
$$

To show that $D^{N} R_{2 N}(W+b)$ is bounded for all $W$ on $C_{1}$ and $C_{2}$, consider the integral

$$
\text { (4.11) } \begin{aligned}
z I & =\int_{C_{W}} \frac{z G(\eta+b)}{\eta^{N+m}(\eta-W)^{N-m+1}} d \eta \\
& =\int_{C_{W}} \frac{1}{\eta^{N+m}(\eta-W)^{N-m+1}\left(e^{V(\eta)}-1\right)} d \eta, \quad m=0,1, \ldots, N .
\end{aligned}
$$


For any fixed point $W$ on $C_{1}$ or $C_{2}$, the contour $C_{W}$ has the points $\eta=$ $0, b, W$ in its interior. We can replace $C_{W}$ by the contours $C_{3}, X Y=\pi$, $Y>0$ and $C_{4}, X Y=-\pi, Y<0$. Let $J$ denote the above integral along the curve $C_{3}, \eta=X+i Y, X Y=\pi, Y>0, d Y=\left(-\pi / X^{2}\right) d X$. Then, (4.12)

$$
|J| \leq M \int_{0}^{\infty}\left(X^{2}+\frac{\pi^{2}}{X^{2}}\right)^{-(N+m) / 2}|X+i Y-W|^{-N+m-1} e^{-X^{2} / 4}\left(\frac{X^{2}+\pi}{X^{2}}\right) d X .
$$

Note that $\left(X^{2}+\pi^{2} / X^{2}\right) \geq 2 \pi$. Let $d$ denote the distance function. If $W$ is on $C_{1}$, then by symmetry, $d\left(W, C_{3}\right) \geq d\left(-b, C_{3} \cup C_{4}\right)$. Since $-b \in \Omega^{\prime \prime}$ and $d\left(\Omega^{\prime \prime}, C_{3} \cup C_{4}\right)=\gamma>0$, it follows that $d\left(W, C_{3}\right) \geq \gamma$. If $W$ is on $C_{2}$, let $W=t,-\sigma \leq t<\infty$, then $|X+i Y-W|^{2} \geq \pi^{2} / X^{2}$. Therefore, for $m=0,1, \ldots, N \geq 2$,

$$
\begin{aligned}
|J| & \leq M^{\prime}(m) \int_{0}^{\infty} \frac{X^{2}+\pi}{X^{4}+\pi^{2}}\left\{\gamma^{-N+m-1}+\left(\frac{X}{\pi}\right)^{N+1-m}\right\} e^{-X^{2} / 4} d X \\
& \leq M^{\prime \prime}(m, \gamma)
\end{aligned}
$$

A similar bound holds for the integral along $C_{4}$. Since $\frac{1}{z}$ is bounded for $z \in \Omega$, by (4.11), $|I| \leq M^{\prime \prime \prime}(m, \gamma)$ for some constant $M^{\prime \prime \prime}$. This completes the proof.

\section{REFERENCES}

1. M. Abramowitz and I. A. Stegun, Handbook of mathematical functions with formulas, graphs and mathematical tables, National Bureau of Standards, Washington, DC, 1964.

2. N. Bleistein, Uniform asymptotic expansion of integrals with stationary point near algebraic singularity, Comm. Pure Appl. Math. 19 (1966), 353-370.

3. J. D. Buckholtz, Concerning an approximation of Copson, Proc. Amer. Math. Soc. 14 (1963), 564-568.

4. E. T. Copson, An approximation connected with $e^{-x}$, Proc. Edinburgh Math. Soc. 3 (19321933), 201-206.

5. D. S. Jones, Asymptotic behavior of integrals, SIAM Rev. 14 (1972), 268-317.

6. F. W. J. Olver, Asymptotics and special functions, Academic Press, New York, 1974.

7. K. Soni and B. D. Sleeman, On uniform asymptotic expansions and associated polynomials, J. Math. Anal. Appl. 124 (1987), 561-583.

8. K. Soni and N. M. Temme, On a biorthogonal system associated with uniform asymptotic expansions, Centre for Mathematics and Computer Science, Amsterdam, Report Am-R8709.

9. N. M. Temme, Special functions as approximants in uniform asymptotic expansions of integrals; A survey, Rend. Sem. Mat. Univ. Politecn. Torino, Special Issue, (1985), 289-317.

10. G. N. Watson, Approximation connected with $e^{x}$, Proc. London Math. Soc. (2) 29 (192829), 293-308.

11. R. Wong, On uniform asymptotic expansion of definite integrals, J. Approx. Theory 7 (1973), 76-86.

Department of Mathematics, University of Tennessee, Knoxville, Tennessee 37996- 